\title{
Review: home visitation by nurses beginning prenatally and extending through infancy prevents child abuse and neglect
}

\author{
MacMillan HL with the Canadian Task Force on Preventive Health Care. Preventive health care, 2000 update: prevention \\ of child maltreatment. CMAJ 2000 Nov 28;163:1451-8.
}

QUESTION: Are interventions aimed at preventing child maltreatment effective?

\begin{abstract}
Data sources
Studies were identified by searching Medline, HealthSTAR, PsycINFO, ERIC, and Current Contents for the years 1993-9 using the content terms child abuse, child neglect, battered child syndrome, incest, prevention and control, and screening combined with the methodological terms statistics and numerical data, aetiology, epidemiology, experimental design, meta-analysis, and literature review. Experts in the field were also consulted.
\end{abstract}

\section{Study selection}

Studies were selected if they were original research articles, reviews, meta-analyses, or practice guidelines that described interventions to prevent child maltreatment.

\section{Data extraction}

The lead author critically appraised the key articles and extracted data on preventive strategies and outcomes. A panel of experts used a consensus process to analyse and discuss the data, and to grade the level of evidence and strength of recommendations according to established methods of the Canadian Task Force on Preventive Health Care.

\section{Main results}

3 studies that assessed screening showed limited ability to predict future maltreatment because of high false positive results. All 4 reviews that examined the effectiveness of perinatal and early childhood programmes in preventing child physical abuse and neglect showed some positive outcomes. 2 randomised controlled trials evaluated home visitation by nurses for first time mothers of low socioeconomic status, single parents, or teenaged parents. 15 years follow up of 1 trial showed reduced child abuse and neglect in the group receiving visits prenatally and throughout infancy. Another trial showed reduced injuries and ingestions at 4 years follow up in the group receiving home visits. 3 trials evaluating the effectiveness of paraprofessional home visitors showed no difference between groups. 1 study evaluated the effectiveness of a comprehensive healthcare programme involving prenatal, postnatal, and paediatric care. No difference was seen between groups for physical abuse or neglect. Because of methodological weaknesses, no conclusions could be drawn from 2 studies, 1 evaluating a parent education and support programme and another evaluating a combined services programme. 2 systematic reviews of educational programmes to teach children strategies to avoid sexual abuse showed improved knowledge and prevention skills but did not necessarily transfer to a reduction in incidence of sexual victimisation.

\section{Conclusions}

The strongest evidence for prevention of child abuse and neglect supports a programme of frequent home visits by nurses beginning prenatally and extending through infancy. Screening to identify those at risk or programmes involving home visits by paraprofessionals are not recommended.

\section{COMMENTARY}

The careful review by MacMillan $e t$ al of the literature on child abuse prevention highlights the importance of understanding how well designed research informs practice in preventing child abuse. In the only physical child abuse prevention intervention with documented long term efficacy, the nurse home visitation programme (NHVP) reduced child abuse over a 15 year period using an intervention that consisted, on average, of only 32 visits. ${ }^{1}$

A number of implications exist for clinicians seeking to lessen their patients' risk of child abuse. Among the primary goals of the NHVP was assistance in building supportive relationships with family members and friends and linking women with needed health and human services-concrete services that can also be addressed by individual mental health providers. The developmental literature shows that parents who perceive themselves as having little power over their lives are more likely to engage in coercive and punitive parenting practices. ${ }^{2}$ It is therefore not surprising that the NHVP was most helpful to those families who at the start of the programme perceived themselves as having the least control over their lives. ${ }^{3}$ In their work with high risk families, one of the most crucial roles clinicians can have is in actively empowering their clients, as did the nurses in the NHVP.

A recent study of the NHVP found that in the $21 \%$ of the sample that reported frequent incidents of domestic violence, the programme did not reduce child maltreatment. ${ }^{1}$ The programme has therefore been modified so that the visiting nurses address domestic violence using proven techniques for reducing risk such as safety planning and conflict resolution skills. In light of this finding, clinicians should carefully assess whether marital violence in high risk families may be compromising their therapeutic efforts. David Pelcovitz, $\mathrm{PhD}$

North Shore University Hospital Manhasset, New York, USA

1 Eckenrode JG, Ganzel B, Henderson CR, et al. Preventing child abuse and neglect with a program of nurse home visitation: the limiting effects of domestic violence. JAMA tation: the limiting

2 Bugental DB, Lyon JE, Krantz J. Who's the boss? Differential accessibility of dominance ideation in parent-child relationships. Journal of Personality and Social Psychology 1997;72:1297-309.

3 Olds DL, Henderson CR, Kitzman HJ, et al. Prenatal and infancy home visitation by nurses: recent findings. Future Child 1999;9:44-65.
Source of funding: Canadian Task Force on Preventive Health Care.

For correspondence: Dr H L MacMillan, Hamilton Health Sciences Corporation, Psychiatry/Pediatrics Department, Center for Studies of Children at Risk, Chedoke Division, 2nd Floor, PO Box 2000, Hamilton, Ontario L8N $3 Z 5$, Canada. Fax +1905 5746665 .

A modified version of this abstract appears in Evidence-Based Nursing. 